\title{
NON-PERTURBATIVE DYNAMICS OF ATOMS IIN STRONG LASER FIELDS: ADIABATIC STABILITY OF HYDROGEN ATOM
}

\author{
F.II.M. Faisal aNd L. Dimou \\ Fakultät für Physik, Universität Bielefeld, 33615 Bielefeld 1, Germany
}

\begin{abstract}
Recent investigations of laser-atom interaction at high intensities have led to the discovery of several unexpected phenomena, and their studies have deepened our understanding of non-perturbative dynamics of quantum systems subjected to unusually strong radiation fields. For the analysis and interpretation of these phenomena. We have developed an $a b$ initio non-perturbative method, the Floquet close-coupling method, and applied it to analyse the highly non-perturbative problem of adiabatic stability of hydrogen atom. $A b$ initio rates of ionization as a function of the laser intensity for the excited circular states as wcll as related non-circular states are obtained. Our quantitatively accurate results clearly show that the predictions of the previous approximate theories such as the well-known "high-frequency" theory are qualitatively similar but differ quantitatively. The analysis of the dependence of adiabatic stability on the principal quantum number, the angular momentum, as well as on the magnetic quantum number for the Rydberg states are complemented by investigations of the behavior of the ground state for frequencies below the ionization threshold. In the latter situation we found the existence of stability windows within which the ionization probability decreases with increasing intensity but outside of which the atom becomes more unstable. It is shown here that the mechanism for the occurrence of stability windows is the self-tuned anti-resonance at specific intensities.
\end{abstract}

PACS numbers: $32.80 . \mathrm{Fb}, 32.80 \mathrm{Wr}$

\section{Introduction}

Investigations of multiphoton processes have been successfully performed in the past using the lowest non-vanishing order perturbation theory (LOPT) [7] which depends, as is well known, on the expansion of the wave function of the total system in powers of the photon-atom coupling parameter (which is proportional to the strength of the field). For non-resonant frequency conditions one expects such an expansion to converge for sufficiently weak fields. For strong fields (even under non-resonant conditions) the higher order terms of the series can be larger than 
the leading term and the perturbation expansion breaks down. It can be easily estimated [7] that this occurs for optical frequencies at intensities greater than about $10^{13} \mathrm{~W} / \mathrm{cm}^{2}$. The present day lasers can reach intensities far above this value and thus have opened up the entire field of investigation of non-perturbative quantum dynamics. In this domain the conceptual edifice, developed through decades of experience with weak or moderately strong light fields; turns out to be unreliable. What is required in this circumstance is a fully non-perturbative method of solving the mathematical problem and a new system of concepts to understand the new phenomena intuitively. Much progress has recently been made in this direction which has clarified much of the mechanisms belind the new phenomena of the so-called "above threshold ionization (ATI)" [8], "above threshold detachment (ATD)" [9-11] and the "multi-harmonic generation (MIIG)" [12, 13]. A number of approximate theories, which have been developed initially before the discovery of some of these non-perturbative phenomena, such as the well-known KFR-theory [5] and the high-frequency approximation [4] have turned out to be very useful in understanding them semi-quantitatively. Furthermore, a number of quantitatively exact new theories have been developed more recently, for example: (a) the theory of direct numerical integration on a space-time grid [14], (b) the Floquet theory on a complex $L^{2}$-basis $[16,17,25]$, (c) the Floquet close-coupling (FCC) theory [1-3, $6,18-20]$ and the closely related Floquet $R$-matrix theory [21]. We note that the class of theories (a) is most useful in the case of short pulsed radiation, whereas the classes (b), (c) and (d) are most useful for adiabatic pulses, for which the pulse duration is much larger than the central period of the field. It is worth noting also that in the class of the Floquet theories (b), the continuum of the total system is treated effectively as a damped $L^{2}$-wave function, whereas in the Floquet close-coupling method (c) or the $R$-matrix method (d), the fully oscillating nature of the continuum wave functions is respected.

In this work we present first the essentials of the ab initio Floquet close-coupling theory, which have been applied recently to strong-field ionization of hydrogen atom $[2,6,17,19]$, detachment of hydrogen negative ion [22], and radiative scattering dynamics $[1,3,20]$ including radiative Rutherford scattering, stimulated resonant-bremsstrahlung, and stimulated capture processes. Here we shall investigate the highly counter-intuitive problem of adiabatic stability. We shall present $a b$ initio results of the rates of ionization of a whole set of excited states of atomic hydrogen as a function of the laser intensity which unambiguously demonstrate the phenomenon of adiabatic stability in hydrogen atom. The results are obtained both for a sequence of circular- and non-circular states.

We analyse the so-called "supra-threshold" case (in this case the frequency is sufficiently large for ionization by absorption of a single photon), and contrast the findings with those for the case of "sub-threshold frequency" (in this case the frequency can be comparable to the excitation energies of the atom so that ionization by absorption of a single photon generally is not allowed). It will be shown that the nature of adiabatic stability in these two frequency domains is very different; the high frequency stability increases monotonically with respect to the intensity while the "sub-threshold frequency" stability can give rise to stability windows on the intensity axis. We shall give a physical interpretation of the latter 
phenomenon which has been observed earlier in several ab inilio calculations [6].

\section{Floquet close-coupling theory}

The Schrödinger equation for the prototype real system of the hydrogen atom interacting with a monochromatic radiation field can be written down in the minimal-coupling gauge as $(e=\hbar=m=1)$ :

$$
\mathrm{i} \frac{\mathrm{d}}{\mathrm{d} t} \Psi(t)=\left[-\frac{1}{2} \Delta+V(|r|)-\frac{\mathrm{i}}{\mathrm{c}} \nabla \cdot A(t)+\frac{1}{2 c^{2}} A^{2}(t)\right] \Psi(t) .
$$

Note that in the present gauge the interaction Iamiltonian for an uniform field leads to a non-vanishing coupling at asymptotically large distances. Note also that the well-known length-gauge interaction Hamiltonian in the uniform field in fact diverges asymptotically. In order to avoid non-vanishing interaction potential at asymptotically large distances we introduce an unitary space-translation transformation [23] on the wave function

$$
\Psi(t) \rightarrow \exp \left[\int^{t} \mathrm{i}\left(\frac{1}{c} p \cdot A(l)-\frac{1}{2 c^{2}} A^{2}(l)\right) \mathrm{d} t\right] \Psi(\ell)
$$

The Schrödinger equation (1) in the space-translated reference frame (also known as the "oscillating frame" or the "K-H frame") takes the useful form

where

$$
\mathrm{i} \frac{\mathrm{d}}{\mathrm{d} t} \Psi(r, t)=\left[-\frac{1}{2} \Delta+V(|r-\alpha(t)|)\right] \Psi(r, t),
$$

$$
V(|\boldsymbol{r}-\alpha(t)|)=-\frac{1}{|\boldsymbol{r}-\alpha(t)|}
$$

in which the Coulomb potential appears shifted in $\boldsymbol{r}$ by the instantaneous "quiver radius"

$$
\alpha_{0}(t)=-\frac{1}{c} \int^{t} \boldsymbol{A}\left(t^{\prime}\right) \mathrm{d} t^{\prime}
$$

where $\boldsymbol{A}(t)$ is the vector potential.

The $(3+1)$-dimensional partial differential equation (3) poses formidable difficulties in solving it directly by numerical means. This is specially so for adiabatically changing pulses which require a very large space-time grid for direct integration. Since most, if not all, of the experimental pulses are of much longer duration than the period of their corresponding carrier frequency, they are effectively switched on and off in an adiabatic fashion during the interaction. The Floquet close-coupling method can handle this situation particularly conveniently in view of the fact that adiabatic pulses lead to an effective periodicity of the Hamiltonian, which is appropriate for the Floquet analysis. The essence of the method is to convert the partial differential Eq. (3) (which remains untractable for the very large space time domain which arises) into an infinite set of ordinary differential equations for the so-called channel wave functions, $F_{n / m}(r)$. This is achieved by expanding the total wave function in Floquet plus spherical partial waves as follows $[1-3,18,19]$ :

$$
\Psi(r, t)=\sum_{n l m} \exp \{-\mathrm{i}[E t-n(\omega t+\delta)]\} \frac{1}{r} F_{n l m}(r) Y_{l m}(\theta, \phi) .
$$


Substituting (6) in (3), equating coefficients of $\exp [i n(\omega t+\delta)]$ on both sides and projecting from the left onto the spherical harmonics, we readily obtain the Floquet close-coupling equations

$$
\left[\frac{\mathrm{d}^{2}}{\mathrm{~d} r^{2}}+k_{n}^{2}-l(l) / r^{2}+\frac{2}{r}\right] F_{n l m}(r)=-2 \sum_{n^{\prime} l^{\prime} m^{\prime}} V_{n l m, n^{\prime} l^{\prime} m^{\prime}}(r) F_{n^{\prime} l^{\prime} m^{\prime}}(r),
$$

where $V_{n l m, n^{\prime} l^{\prime} m^{\prime}}(r)$ are the channel coupling potentials [1, 3] which arise from the matrix elements, of the time-Fourier components of the interaction potential (4), between the spherical harmonics. The set of quantum numbers $\{n l m\}$ defines a reaction channel in which the electron finds itself in the angular momentum states $(l m)$ having absorbed or emitted $n$ photons from the initial state. It is worth noting that the full set of equations (7) is exact and reduces to the time averaged (high frequency) approximation [4] when the off-diagonal couplings in $n$ are neglected. We remark also that an essential difference of the present method with respect to the $L^{2}$-methods lies in the fact that while in the latter method each $l$-state must be approximated by a whole set of radial basis states, in the present case a single l-channel accounts for all the radial states of the same symmetry automatically through the solution of the channel radial differential equation. For the problem of decay of a bound state which occurs during ionization or detachment processes, the system of Eqs. (7) can be solved most conveniently by imposing $[2,22]$ Siegert's outgoing wave boundary conditions in the open channels (i.e. for which $\operatorname{Re}(E-n \omega)>0$ ), and exponentially decreasing asymptotic boundary conditions in the closed cliannels (i.e. for which $\operatorname{Re}(E-n \omega)<0$ ). This is implemented by integrating the coupled system of Eqs. (7) from the origin outward and from the asymptotic region (where the channel solutions decouple and go over to the Coulomb waves with respective channel wave numbers) inward and matching the logarithmic derivative-matrix of the inward and outward propagated solutions at an intermediate radius, $r_{\mathrm{m}}$. This leads to the energy determining secular equation

$$
\operatorname{det}\left|L_{\text {in }}\left(r_{\mathrm{m}}\right)-L_{\text {out }}\left(r_{\mathrm{m}}\right)\right|=0,
$$

where the log-derivative matrix $L(r)$ is defined by $L(r)=(\mathrm{d} / \mathrm{d} r) F(r) / F(r)$.

Perhaps the most important property of the Floquet close-coupling theory lies in the finite nature of the distance (say, $r_{a}$ ) of the border of the asymptotic region at which the channel solutions connect analytically to the Coulomb wave functions in all channels. (The distance $r_{a}$ can be determined by the smallness of the off-diagonal coupling potentials, $\mathrm{O}\left(1 / r^{2}\right)$, to the diagonal potential $\mathrm{O}(1 / r)$, to any given accuracy.) This has the remarkable effect of generating the wave function of the system, which extends analytically to infinity along the radial axis. In other words, the FCC-method tackles the cardinal problem of integration over an infinite radial domain, on a finite machine, by combining the numerical integration up to a finite distance, $r_{a}$, with the analytical properties of the system.

Equation (8) has solutions, in general, in the complex $E$-plane; this is due to our imposition of Siegert's outgoing wave (complex) open-channel boundary conditions. From the general $S$-matrix theory of resonance follows the interpretation of the complex eigenvalues of the Siegert's determinant (8): the imaginary part of the $\lambda$-th complex eigenvalue, whose real part is connected to the unperturbed eigenvalue of the initial state (in the limit of vanishing field strength), gives 
the decay rate $\Gamma$ of that state: $\Gamma=-2 \operatorname{Im}\left(E_{\lambda}\right)$; the real part itself provides the "quasi-energy" of the associated Floquet (or the "dressed")-state of the system.

\section{Ab initio results of adiabatic stability in hydrogen atom}

\subsection{Stability behavior of circular and non-circular states: "supra-threshold" case}

According to the "high-frequency" approximation [4], i.e. in the limit in which the laser frequency is very large compared to the perturbed binding energy of the initial state, the adiabatic rate of ionization can be estimated, in the space-translated frame of reference, by considering only the time averaged interaction. Since all the time dependent components of the interaction potential average out to zero, only that part which is constant in time determines the eigenstates of the system in the average potential, which are, of course, stable in the zeroth approximation. According to this model, the adiabatic rates of ionization of the initial state of the system is then induced by the perturbation of the neglected oscillating terms, in the first approximation. Detailed calculations using this model show [4] a decrease, instead of increase, in the ionization rates with intensity, beyond a certain critical value. This so-called adiabatic stability phenomenon is highly counter-intuitive and has no analogy in the usual response of the atom to weak light fields.

For the experimental observation of the phenomenon it is required that the atom is not completely ionized at the lower intensities (during the rise of the adiabatic pulse), before the stability region at higher intensities is reached. For a long time it was popularly believed that neutral atoms could not be subjected to arbitrarily strong fields because of this so-called "pre-ionization saturation" effect. The adiabatic stability phenomenon provides a counter example to this popular belief, since if the pre-ionization rates at lower intensities are sufficiently low until the stability region is reached, then the atom could experience any higher intensity (without leading to ionization-saturation). Choice of suitable initial states e.g. the so-called circular states (for which the $(l, m)$-quantum numbers are the maximum allowed ones in the $n$-th shell) of the atom, which are expected to keep the total ionization rate at lower intensities sufficiently small, could then permit one to observe the phenomenon experimentally. In fact, it suffices to choose pulse durations $r$ such that $\Gamma_{\mathrm{m}} \tau \ll 1$, where $\Gamma_{\mathrm{m}}$ is the maximum rate of ionization, in order to be able to subject the atom to arbitrarily high peak intensities. Very recently the Amsterdam group [24] has reported the first observation of adiabatic stability phenomenon in the case of $5 g(m=4)$ state in the complex Ne atom. For the preparation and analysis of experiments on the fundamental system of hydrogen atom [25] (we may note here that currently experiments are under way in Bielefeld University, and in Brookhaven National Laboratory (under the Brookhaven-Bielefeld experimental collaboration) to this end) it is desirable to have accurate prediction of the dependence of the rates of ionization on the intensity of the field, in particular, the maximum ionization rates (or the minimum "lifetime" against ionization) and the corresponding critical values of the peak intensity. It is also of interest to determine the dependence of these parameters on the quantum numbers of the initially 
prepared circular and non-circular states for currently available lasers. We present below results of our theoretical investigations for the same.

Figure 1 shows the rate of ionization for the initially prepared circular Rydberg state: $(n=5, l=4, m=4)$ for the well-known Nd laser at the fundamental wavelength $\lambda=1064 \mathrm{~nm}$. It is seen clearly that the adiabatic stability phenomenon

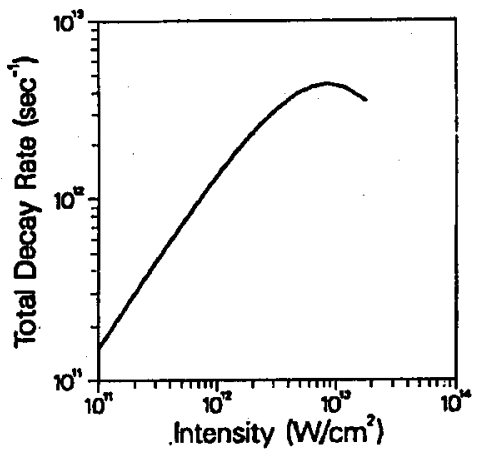

Fig. 1. Ionization rates of the circular Rydberg state $(n=5, l=4, m=4)$ of hydrogen atom vs. the peak intensity of $\mathrm{Nd}$ laser $(\lambda=1064 \mathrm{~nm})$.

occurs in this case beyond the critical intensity $I_{\mathrm{c}}=0.856 \times 10^{13} \mathrm{~W} / \mathrm{cm}^{2}$, which is only of moderate strength in the atomic scale. The maximum ionization rate in this case is $\Gamma_{\mathrm{m}}=4.46 \times 10^{12} / \mathrm{s}$ (lifetime $\tau=1 / \Gamma=224 \mathrm{fs}$ ). This would require a pulse duration smaller than $0.1 \mathrm{ps}$ to observe the phenomenon.
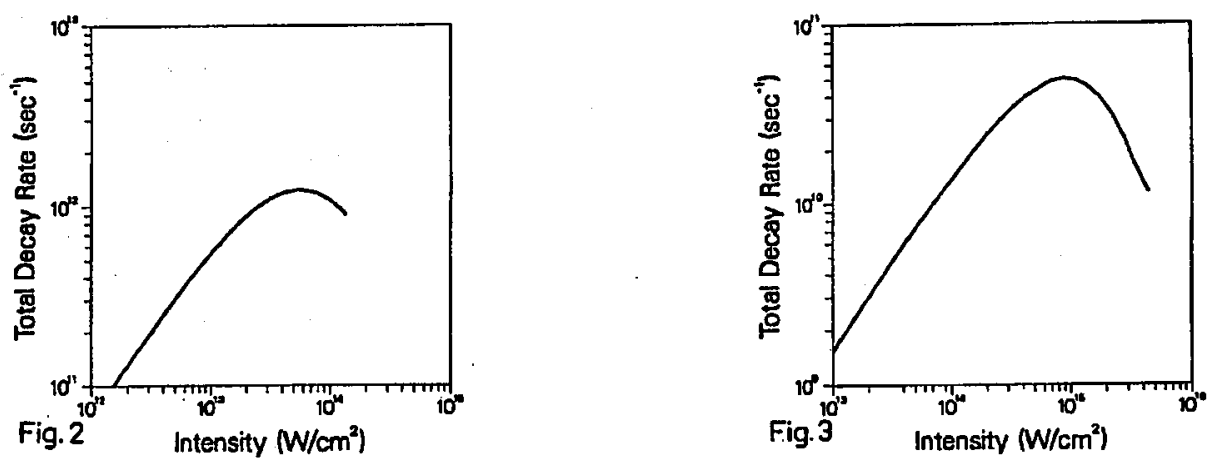

Fig. 2. The same as in Fig. 1 but $\lambda=620 \mathrm{~nm}$.

Fig. 3. The same as in Fig. 2 but for $\lambda=248 \mathrm{~nm}$.

In Figs. 2 and 3 we show the adiabatic stability results for the same circular state as in Fig. $1((n, l, m)=(5,4,4))$ for two other currently available laser wavelengths, namely, $620 \mathrm{~nm}$ and $248 \mathrm{~nm}$, respectively. It is seen that the maximum rates of ionization are $\Gamma_{\mathrm{m}}=1.21 \times 10^{12} / \mathrm{s}$ (lifetime $\tau=0.81 \mathrm{ps}$ ), and 
$\Gamma_{\mathrm{m}}=5.36 \times 10^{10} / \mathrm{s}$ (lifetime $\tau=19 \mathrm{ps)}$, respectively. The corresponding critical intensities are $I_{\mathrm{c}}=5.52 \times 10^{13} \mathrm{~W} / \mathrm{cm}^{2}$ and $I_{\mathrm{c}}=9.14 \times 10^{14} \mathrm{~W} / \mathrm{cm}^{2}$, respectively. They should clearly permit reaching the stability region with pulse durations in the region of 1.0 to $0.1 \mathrm{ps}$. In fact, the first experimental observation of the phenomenon of adiabatic stability has been reported by the FOM-Amsterdam group [24], for the same initially prepared (one electron)-state of Ne atom, and using the same $620 \mathrm{~nm}$ sub-picosecond laser. Their experimental result in $\mathrm{Ne}$ is consistent with the quantitative results for hydrogen calculated here and that calculated using the $L^{2}$-basis method [25]. It is worth noting that the methods of the elaborate calculations and the gauges used by the last authors and by us are entirely different; hence the close agreement between these two sets of $a b$ initio calculations speaks strongly in favor of the quantitative reliability of the same results.
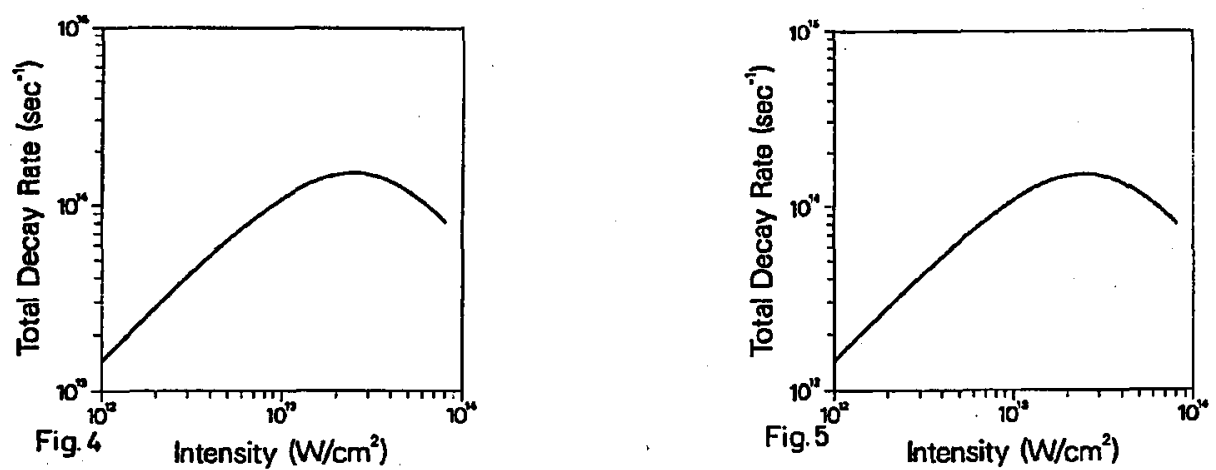

Fig. 4. Ionization ratcs of the circular state $(n=4, l=3, m=3)$ of hydrogen atom vs. the peak intensity of the $620 \mathrm{~nm}$ laser.

Fig. 5. Ionization rates of the circular $(n=3, l=2, m=2)$-state of hydrogen atom vs. the peak intensity of the $620 \mathrm{~nm}$ laser.

We now consider the problem of adiabatic stability for the lower lying circular states as well. Figures 4 and 5 show the rates of ionization as function of the intensity, for the $620 \mathrm{~nm}$ laser, for the initial states $(n, l, m)=(4,3,3)$ and $(3,2,2)$, respectively. It is clear from these two figures that the phenomenon of adiabatic stability occurs for these circular states as well, but the maximum rates are an order of magnitude or more larger than in the case of the circular $(5,4,4)$-state (c.f. Fig. 2). Thus, observation of the stability behavior for these states would require at present unrealistically short pulse durations.

It is clear from the results presented in the above figures that preparation of the hydrogen atom in the circular state $(n=5, l=4, m=4)$ (by a four-photon resonant excitation process with a circularly polarized laser, e.g. as in the FOM-Amsterdam experiment with $\mathrm{Ne}$ ) is sufficient to provide a good opportunity to demonstrate the phenomenon of adiabatic stability in the fundamental hydrogen atom with the currently available $620 \mathrm{~nm}$ laser.

It is interesting to ask further how the departure from circularity of the initial 
state may affect the rate of ionization and the problem of stability. Answer to this question can be found from the results shown in Figs. 6 and 7 which correspond to the non-circular initial states $(n, l, m)=(5,4,3)$ and $(5,3,3)$, respectively (for the $620 \mathrm{~nm}$ laser). It can be seen that the stability phenomenon again occurs but
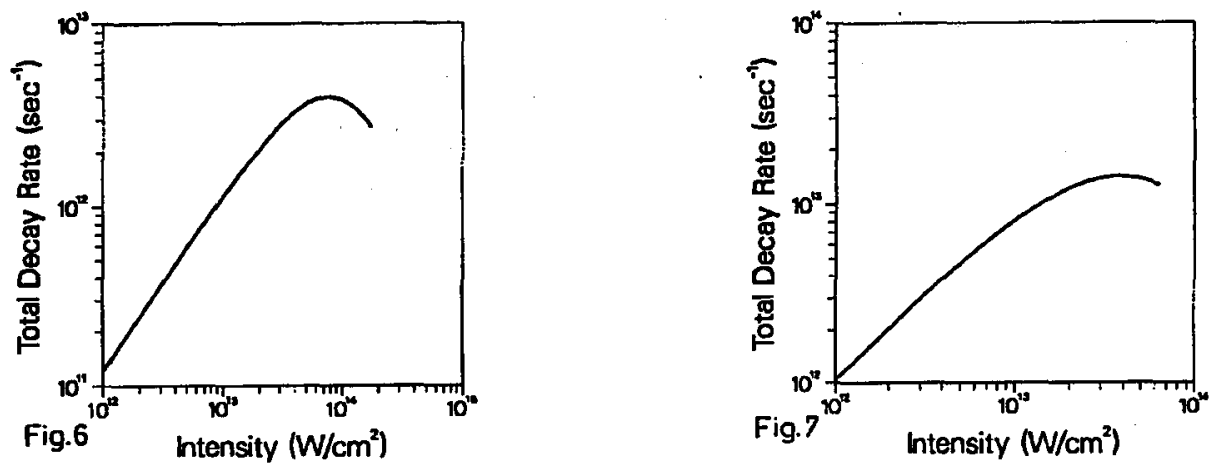

Fig. 6. Ionization rates of the non-circular $(n=5, l=4, m=3)$-state of hydrogen atom vs. the peak intensity of the $620 \mathrm{~nm}$ laser.

Fig. 7. Same as in Fig. 6 but for the state $(n=5, l=3, m=3)$.

the maximum rates of ionization in these cases are significantly higher than that of the circular states with the same principal quantum number.

The lower values of the maximum ionization rates of the circular states are consistent with the physical picture that a circular state when driven perpendicular to the plane of the "orbit" (along the direction of polarization of the linearly polarized ionizing field) interacts less strongly with the nucleus than the non-circular states whose "orbits" are oriented obliquely to the polarization direction. The former state gets less chance of transferring the momentum to the nucleus, which is necessary for the photon absorption, than the latter and hence ionizes less. One, therefore, can expect that preparation of the higher lying circular states (e.g. $(n, l, m)=(6,5,5)$ - or $(7,6,6)$-states $)$ as initial states, will also serve in achieving the conditions for the experimental test of the adiabatic stability phenomenon in $\mathrm{H}$ atom.

Another special characteristic of the circular states is worth mentioning in this context. This is the effective ground-state like character of any circular state, with respect to the linearly polarized light, and the associated stored excitation energy at the end of the pulse. Thus, due to the dipole selection rule, $\Delta m=0$, there cannot be any dipole coupling to any state of the hydrogen atom which lie on or below the shell of the excited circular state of interest. This is because such lower lying states have their maximum magnetic quantum number smaller than that of the circular state of interest. This is also a reason why the lifetime against spontaneous radiation tends to be large for the circular states, compared to the non-circular states. In the presence of a linearly polarized field, these facts open up an alternative to the usual possibility of finding the signature of stabil- 
ity by measuring the total number of ions or electrons. Thus, one may use the complementary method of detecting the number of neutrals left undestroyed in the initially prepared state as a function of intensity. The excited circular state $(5,4,4)$, or those lying near it, will have enough stored excitation energy to be detected (e.g. by surface ionization signal), at the end of the stabilizing pulse.

\subsection{Nature of adiabatic stability for frequencies below the ionization threshold}

We now investigate the problem of adiabatic stability for frequencies which are not large enough to cause ionization by one-photon absorption. It has been noted in time-dependent short pulse calculations [14, 15], that for frequencies smaller than the ionization threshold the probability of ionization could became less than unity and it could decrease further with increasing intensity, indicating existence of stability behaviors in the sub-threshold frequency. Subsequent three-dimensional studies with adiabatic fields $[6,17]$ have revealed windows of intensities where adiabatic-stability behavior could be seen to occur. Below we present typical results for the occurrence of such behavior in the sub-threshold frequency regime and conclude this paper with an investigation of the mechanism behind the occurrence of such stability windows. In this paper we obtain an exact numerical solution of this problem.
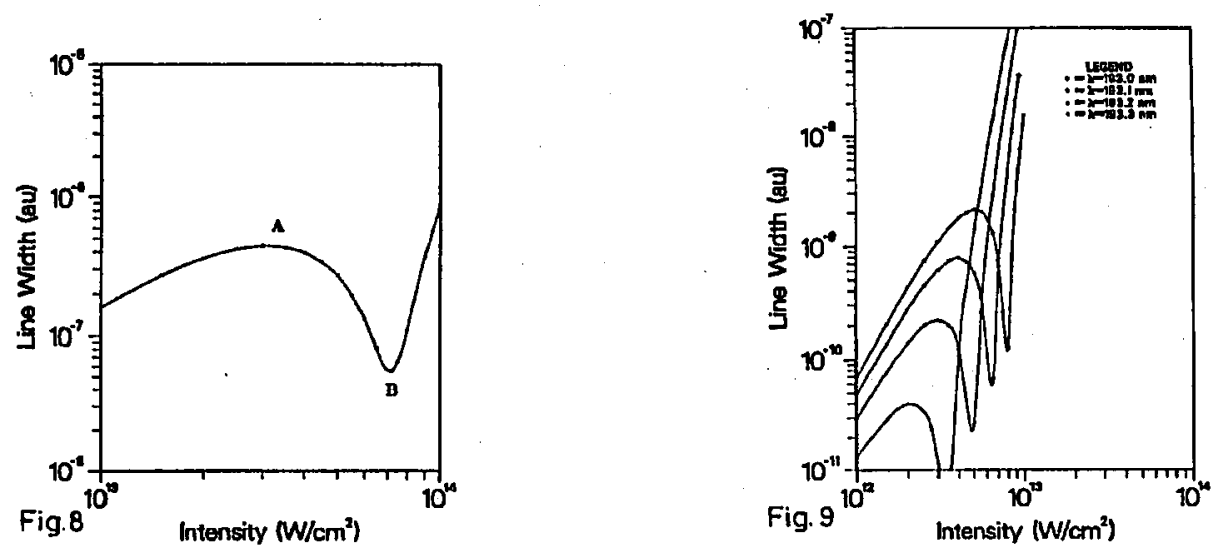

Fig. 8. Line width (ionization rate) of the ground state of II atom vs. intensity, for $\lambda=117 \mathrm{~nm}$, showing a stability window, in the intensity range between $A$ and $B$.

Fig. 9. Sensitive dependence of the stability window on small change of frequency near the wavelength of the excimer laser at $\lambda=193.0,193.1,193.2$, and $193.3 \mathrm{~nm}$, showing the self-tuning of the anti-resonance at the intensities corresponding to the "stability minima".

Figure 8 shows the dependence of the rate of the ground state of $U$ atom as a function of intensity at the wavelength $\lambda=117 \mathrm{~nm}$ (which falls in the domain between the first and the second excited shells of the II atom). It is seen that the rate 
of ionization increases at first with intensity but then reaches a maximum at the point $A$, and begins to decrease with intensity, as with the onset of stability. But unlike in the "supra-threshold" case it does not decrease indefinitely and arrives at a minimum at the point $B$, beyond which it begins to rise again with increasing intensity, leaving behind a "hole" at $B$. Thus in the present case there is only a "stability window" in the intensity domain between the points $A$ and $B$. The stability window is a dual of the anti-resonance phenomenon; the latter phenomenon is known to arise for weak field ionization at a fixed intensity when the frequency is scanned [26]. In the latter context the anti-resonance is known to be due to the destructive interference (which arise during the frequency tuning) between the dominant quantum paths (i.e. perturbative sub-amplitudes) leading to the ionization continuum. The important difference in the present case is that we are dealing with a fixed frequency phenomenon for which the destructive interference is now self induced by dynamic Stark tuning into the anti-resonance.

A testable consequence of this mechanism is that if the frequency is slightly changed then the dynamic Stark tuning will again lead to the anti-resonance minimum, but at a somewhat different intensity. To check the last point we have investigated the rate of ionization of the ground state hydrogen at a number of slightly different wavelengths near that of the well-known excimer laser $(193 \mathrm{~nm})$. Figure 9 shows the ionization rates as a function of increasing intensity for the wavelengths $\lambda=193.0,193.1,193.2$, and $193.3 \mathrm{~nm}$. It is seen that, in fact, in all cases the expected stability minima arise and indeed they arise at somewhat shifted intensities, as required by the mechanism of dynamic Stark tuning of the anti-resonance. In view of this mechanism we may safely predict that for sub-threshold frequencies the stability windows should occur whenever the intensity is strong enough to force the "anti-resonances" into existence, and that it will occur for all atoms subjected to analogous field conditions.

\section{Acknowledgments}

We thank Prof. D. Feldmann for fruitful discussions. This work was partially supported by the Deutsche Forschungs Gemeinschaft.

\section{References}

[1] L. Dimou, F.H.M. Faisal, Phys. Rev. Lelt. 29, 872 (1987).

[2] L. Dimou, F.H.M. Faisal, Phys. Rev. A 46, 4442 (1992).

[3] L. Dimou, F.H.M. Faisal, Laser Phys. 3, 440 (1993).

[4] M. Pont, N.R. Walet, M. Gavrila, Phys. Rev. A 41, 447 (1990).

[5] L.V. Keldysh, Sov. Phys. JETP 20, 1307 (1965); F.H.M. Faisal, J. Phys. B 5, L89 (1973); H.R. Reiss, Phys. Rev. A 22, 1786 (1980).

[6] L. Dimou, F.H.M. Faisal, Phys. Lett. A 171, 211 (1992).

[7] F.H.M. Faisal, Theory of Multiphoton Processes, Plenum Press, New York 1987, Ch. 6.

[8] P. Agostini, F. Fabre, Phys. Lett. A 171, 211 (1979); P. Kruit, J. Kimman, van der Wiel, J. Phys. B 14, L597 (1981). 
[9] C. Blondel, M. Crance, C. Delsart, A. Giraud, J. Phys. B 24, 3575 (1991).

[10] H. Stapplefeld, P. Balling, C. Brink, H.K. Haugen, Phys. Rev. Lell. 67, 1731 (1991).

[11] M.D. Davidson, H.G. Muller, H.B. van Linden van den Ilauvel, Phys. Rev. Lelt. 67, 1713 (1991).

[12] M. Ferray, A. L'Huillier, X.F. Li, L.A. Lompré, G. Mainfray, C. Manus, J. Phys. B 21, L31 (1988).

[13] A. McPherson, G. Gibson, H. Jara, U. Johann, T.S. Luk, I. McIntyre, K. Boyer, R.K. Rhodes, J. Opt. Soc. Am. B 4, 595 (1987).

[14] K. Kulander, Phys. Rev. A 36, 2726 (1987); J. Javanainen, J.II. Eberly, Q. Su, Phys. Rev. A 38, 3430 (1988).

[15] Q. Su, J.H. Eberly, J. Javanainen, Phys. Rev. Lelt. 64, 862 (1990); M.V. Fedorov, M.Yu. Ivanov, J. Opt. Soc. Am. B 7, 569 (1990); K. Kulander, K.J. Schafer, J.L. Krause, Phys. Rev. Lett. 66, 2601 (1991); Q. Su, J.H. Eberly, Phys. Rev. A 43, 2472 (1991); K. Burnett, P.L. Knight, B.R. Piraux, V.C. Reed, Phys. Rev. Lelt. 66, 301 (1991); M. Pont, R. Shakeshaft, Phys. Rev. A 44, R4110 (1991).

[16] A. Maquet, S.I. Chu, W.P. Reinhardt, Phys. Rev. A 27, 2946 (1983); S.I. Chu, K. Wang, E. Layton, J. Opt. Soc. Am. B 7, 425 (1990).

[17] M. Dörr, R.M. Potvliege, B. Proulx, R. Shakeshaft, Phys. Rev. A 43, 3729 (1991); R.M. Potvliege, R. Shakeshaft, Phys. Rev. A 40, 3061 (1989).

[18] A. Giusti-Suzor, P. Zoller, Phys. Rev. A 11, 5178 (1987).

[19] P. Marte, P. Zoller, Phys. Rev. A 43, 1512 (1991).

[20] A. Franz, H. Klar, J.T. Broad, J.S. Briggs, J. Opt. Soc. Am. B 7, 545 (1990).

[21] P.G. Burke, F. Franken, C.J. Joachain, Europhys. Lett. 13, 617 (1990); P.G. Burke, F. Franken, C.J. Joachain, J. Phys. B 24, 761 (1991); M. Dörr, M. Terao-Dunseth, J. Parris, C.S. Noble, P.G. Burke, C.J. Joachain, J. Phys. B 25, 2809 (1992).

[22] L. Dimou, F.H.M. Faisal, in: Super Inlense Laser-Alom Physics, Ed. B. Piraux, Plenum Press, New York 1993.

[23] W. Pauli, M. Fierz, Nuovo Cimento 15, 167 (1938); II.A. Krammers, in: Collected Scientific Papers, North-Holland, Amsterdam 1956, p. 262; W. Henneberger, Phys. Rev. Lett. 21, 838 (1968); F.H.M. Faisal, J. Phys. B 5, L89 (1973).

[24] M.P. de Boer, J.H. Hoogenraad, R.B. Vrijen, L.D. Noordam, I.G. Muller, Plıys. Rev. Lett. 71, 3263 (1993).

[25] R.M. Potvliege, H.G. Smith, Phys. Rev. A 48, R46 (1993).

[26] J. Morrelec, D. Normand, G. Mainfray, C. Manus, Phys. Rev. Lett. 44, 1394 (1980). 